\title{
Recording of multiple holograms in photopolymer films
}

\author{
Kevin Curtis and Demetri Psaltis
}

\begin{abstract}
Multiple images were recorded in DuPont HRF-150 photopolymer as transmission holograms by using angle multiplexing. Agreement with Kogelnik's two-wave diffraction theory for phase gratings, the time considerations and its effect on dynamic range, the necessity of a pre-illumination pulse, and good image quality at readout are reported.

Key words: Holography, photopolymer, optical storage.
\end{abstract}

\section{Introduction}

Angularly multiplexed holograms have potential applications as optical memories, ${ }^{1}$ information processors, ${ }^{2}$ and interconnections. ${ }^{3}$ Important considerations include sensitivity, resolution, and the ability to record permanent holograms. DuPont's photopolymer HRF-150 has already been shown ${ }^{4}$ to have excellent sensitivity and resolution for transmission holograms recorded with blue-green light. With this in mind, we show that HRF-150 is a good candidate for a practical, optical storage medium in which multiple holograms can be recorded. We report the experimental demonstration of multiple holograms recorded in HRF-150.

\section{Experiment}

Ten images were recorded by using the geometry shown in Fig. 1. A sheet of DuPont HRF-150 photopolymer was taped to a glass plate with a centered window cut out of it and mounted on a rotation stage. The holograms were recorded with visible light $(\lambda=488 \mathrm{~nm})$ with a $90^{\circ}$ angle between the writing beams and then fixed with UV light. First, the angular selectivity of the material was determined at $488 \mathrm{~nm}$. The material was $\sim 38 \mu \mathrm{m}$ thick and had angular selectivity (half-width measured at the first minimum) of $1^{\circ}$, which is in good agreement with Kogelnik's two-wave theory ${ }^{5}$ for diffraction from thick

The authors are with the Department of Electrical Engineering, California Institute of Technology, Mail Stop 116-81, 1201 East California Boulevard, Pasadena, California 91125. K. Curtis is also with Northrop, B-2 Division, Pico Rivera, California 90660.

Received 21 February 1992.

0003-6935/92/357425-04\$05.00/0.

(1) 1992 Optical Society of America. phase gratings. The equation for the diffraction efficiency from an unslanted lossless grating is

$$
\eta=\frac{\sin ^{2}\left(\nu^{2}+\xi^{2}\right)^{1 / 2}}{\left(1+\xi^{2} / \nu^{2}\right)}
$$

where

$$
\begin{aligned}
& \nu=\pi \Delta n d / \lambda \cos \theta, \\
& \xi=\left[K \cos (\pi / 2-\theta)-K^{2} \lambda / 4 \pi n\right] d / 2 \cos \theta .
\end{aligned}
$$

In the above equations, $\eta$ is the diffraction efficiency, $\Delta n$ is the index perturbation, $n$ is the index of the material, $\lambda$ is the wavelength, $\theta$ is the angle inside the material measured from the normal to the surface, $K$ is the grating vector's magnitude, and $d$ is the interaction length. Good agreement between experiment and theory for a single plane-wave hologram recorded with a $90^{\circ}$ separation between beams outside the material $\left( \pm 45^{\circ}\right.$ from the normal to the film surface) is shown in Fig. 2. The theoretical plot was calculated using $d=39 \mu \mathrm{m}, n=1.525, K=1.82 \times$ $10^{7} \mathrm{rad} / \mathrm{m}, \Delta n=1.51 \times 10^{-3}, \theta_{\text {Bragg }}=27.62^{\circ}$, and $\lambda=$ $488 \mathrm{~nm}$. The angles were adjusted to account for refraction at the surfaces. The agreement of the experiment and theory indicates two things. First, it indicates that the gratings are not due to surface deformations, which has been observed in similar materials $^{6}$ for low spatial frequency holograms. Also, it indicates that the recorded hologram is most likely a phase grating rather than an absorption grating since the diffraction efficiency of $17.1 \%$ would be too large for an absorption grating.

Figure 3 shows the diffraction efficiency for ten holograms of the same image stored at ten different angular settings of the recording medium while the 


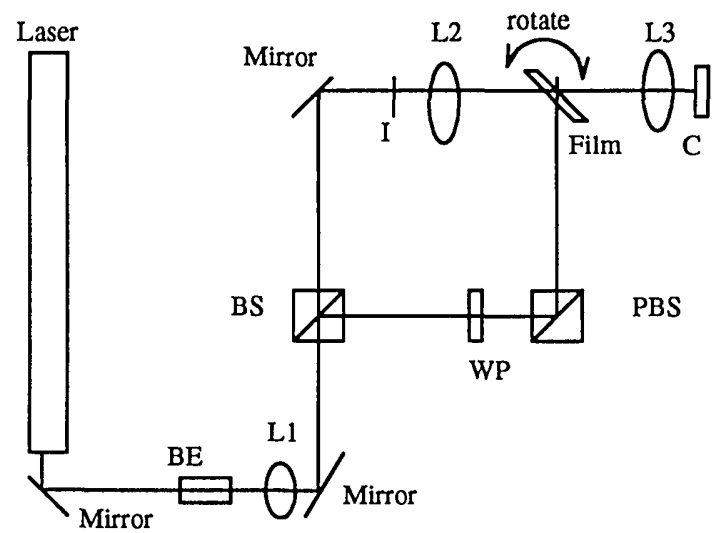

Fig. 1. Setup geometry: BE, beam expander; L1, lens; BS, beam splitter; L2, L3, imaging lenses; WP, half-wave plate; PBS, polarizing BS; I, image; C, camera.

angles between the recording beams are kept the same. The holograms were recorded at intervals of $2^{\circ}$ or at the second minimum of the angular selectivity curve of the adjacent holograms. The holograms were read out by blocking the object beam and by detecting the reconstructed image while rotating the sample. Notice that the first two holograms (at $-10^{\circ}$ and $-8^{\circ}$ ) are weaker than the others, which indicates that exposure to light enhances the sensitivity. Figure 4 shows ten image holograms of roughly equal strength that were recorded with a 5-s preillumination pulse with $1.85 \mathrm{~mW} / \mathrm{cm}^{2}$ intensity $10 \mathrm{~s}$ before the first hologram was recorded. The holograms in Figs. 3 and 4 were recorded by using the setup in Fig. 1; except for the pre-illumination pulse, identical recording parameters were used. The total recording energy per hologram was $1.88 \mathrm{~mJ} / \mathrm{cm}^{2}(1-\mathrm{s}$ exposure per hologram) with an object-to-referencebeam ratio of approximately 1:4.6. The time between holograms is $10 \mathrm{~s}$ for a total experiment run

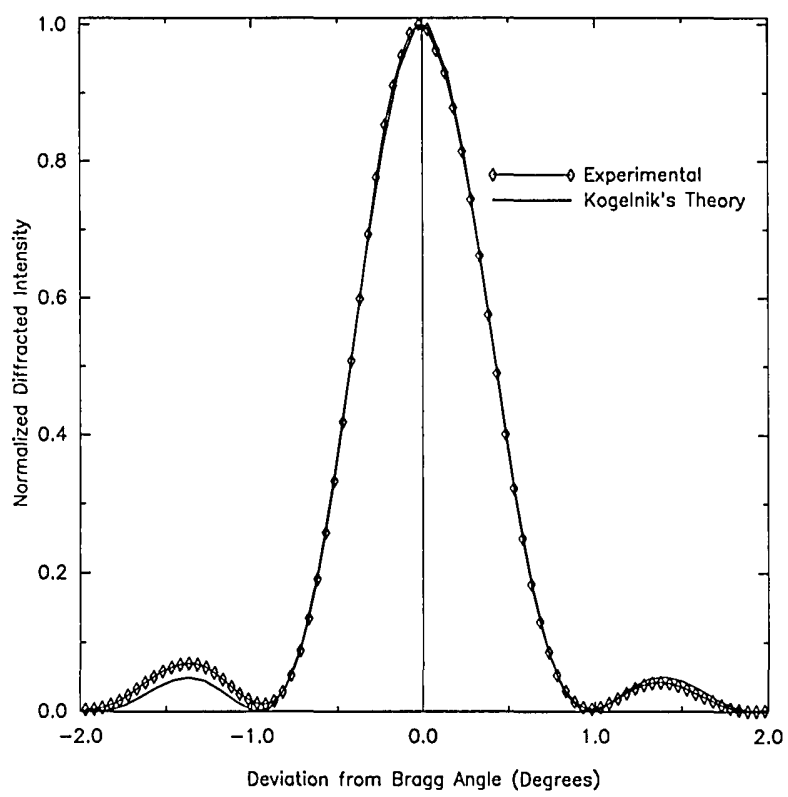

Fig. 2. Angular solectivity of plane-wave hologram.

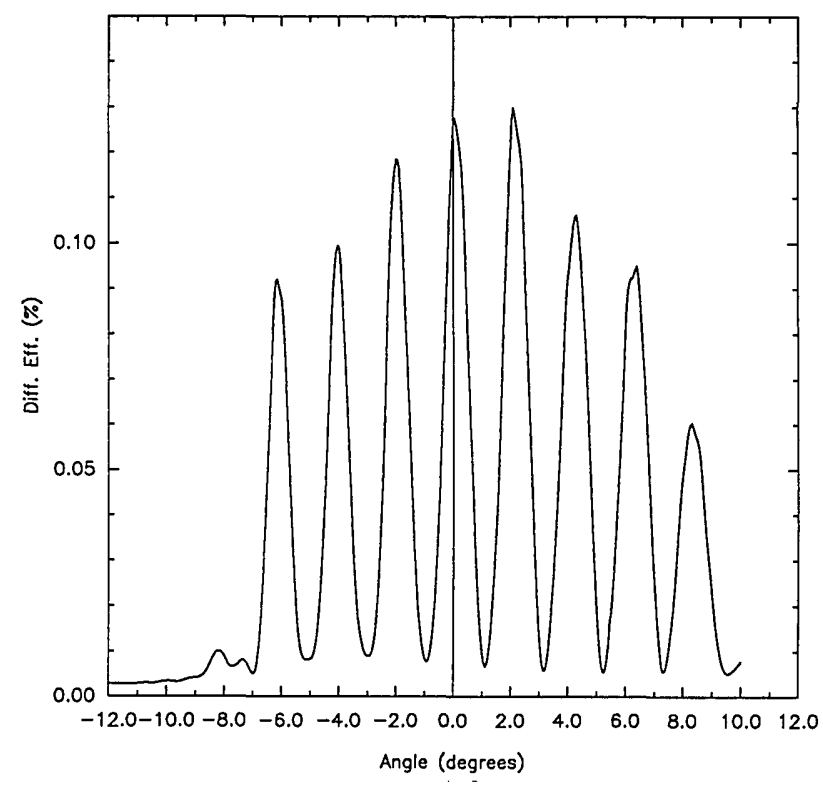

Fig. 3. Ten holograms, no pre-exposure.

time of $120 \mathrm{~s}$. The diffraction efficiency shown is calculated by dividing the diffracted light by the incident-minus-reflected-light intensities and multiplied by 100 to convert to a percentage. The preillumination pulse sensitizes the material and is necessary if the first hologram is to be recorded effectively. Since the material has a finite dynamic range, too long a pre-illumination pulse wastes dynamic range, which causes the later holograms to be weaker than previous ones. Too short of a preillumination pulse fails to sensitize the material, and the first few holograms are lost, as shown in Fig. 3.

The optical quality of the material is good, and Fig. 5 shows a reconstructed image (hologram 6 at $0^{\circ}$ in Fig. 4) with no noticeable distortion. Figure 6 shows the index perturbation versus exposure and demon-

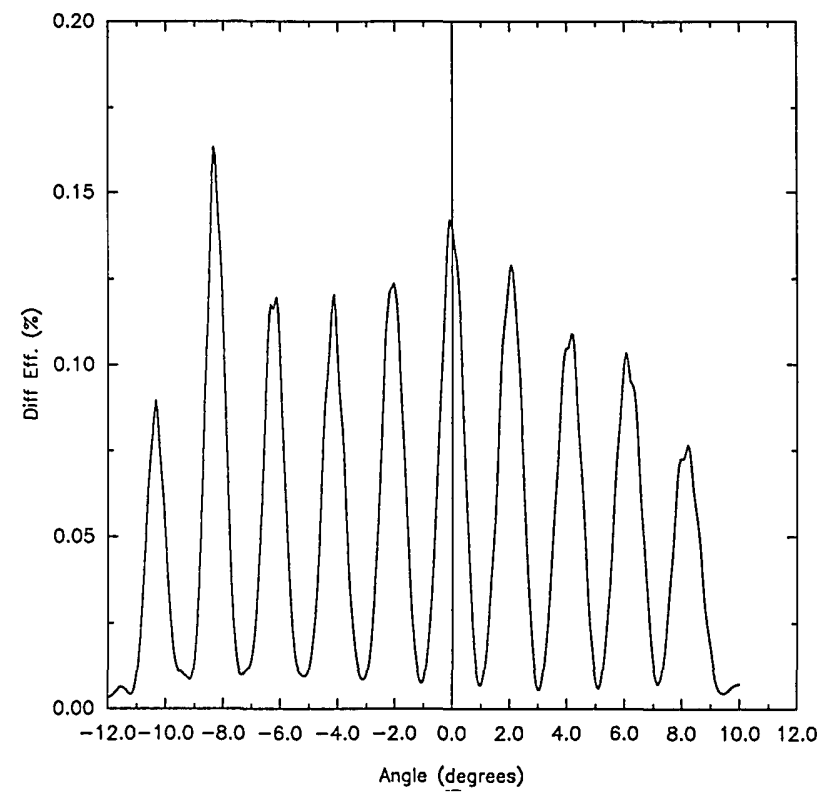

Fig. 4. Ton image-plane holograms. 


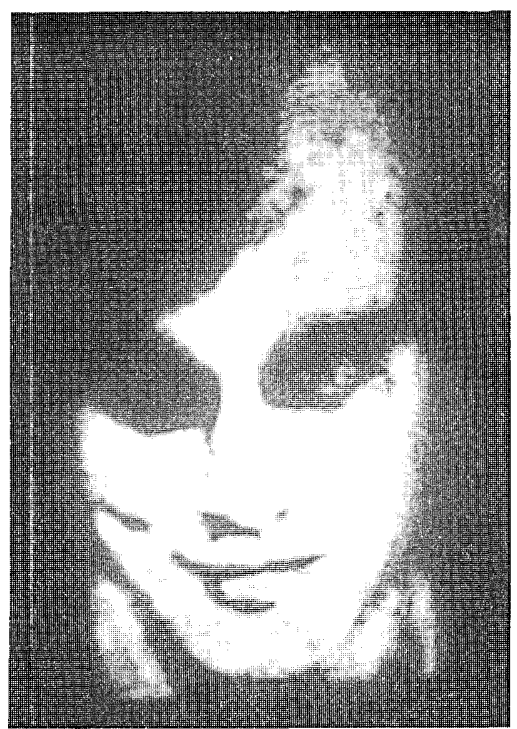

Fig. 5. Reconstructed image.

strates that the pre-illumination pulse does use up dynamic range and increases the sensitivity for small exposures. The index perturbation was calculated by measuring the Bragg-matched diffraction efficiency $(\xi=0)$ and by solving for $\Delta n$ in Eq. (1). In this case the diffraction efficiency used for $\eta$ is diffracted light divided by transmitted light intensity, which eliminates the effects of reflection from the surface and absorption. Notice also that the saturation exposure was $\sim 20 \mathrm{~mJ} / \mathrm{cm}^{2}$; therefore each of the ten holograms should be exposed at $\sim 2 \mathrm{~mJ} / \mathrm{cm}^{2}$. This is close to the actual value of $1.88 \mathrm{~mJ} / \mathrm{cm}^{2}$ per hologram used. Without the preillumination pulse the maximum $\Delta n$ obtained was $\Delta n \approx 0.0028$. The $\Delta n$ we measured is lower than that reported in Ref. 4 $(\Delta n \approx 0.008)$, most likely because of the much higher spatial frequency recorded in our experiment ( 2897

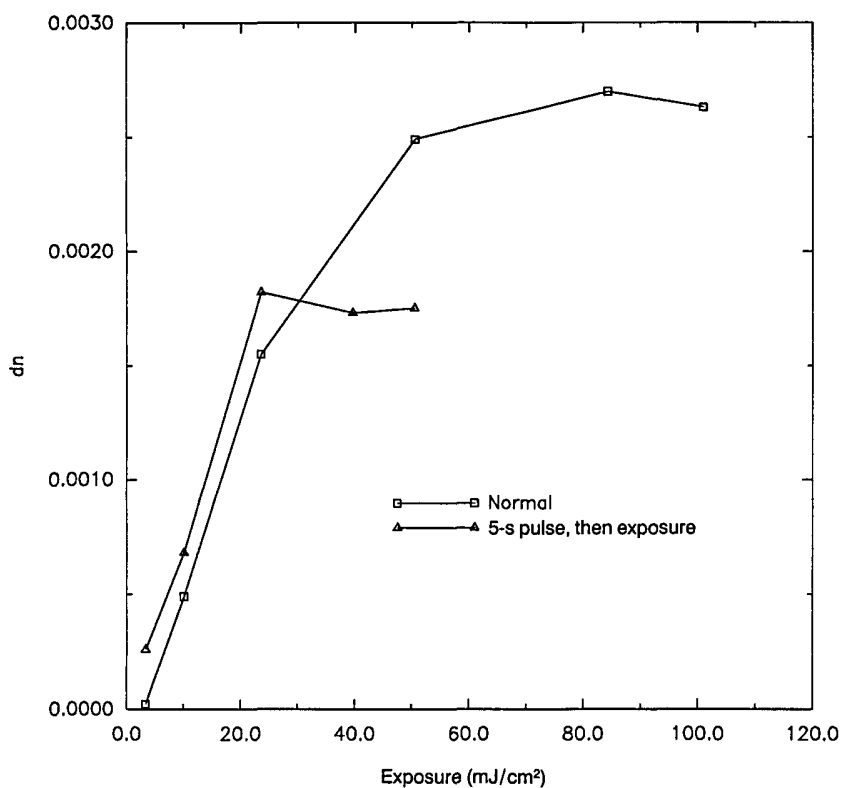

Fig. 6. Index perturbation versus exposure. cycles $/ \mathrm{mm}$ versus 1007 cycles $/ \mathrm{mm}$ ). With the preillumination pulse the saturation exposure for this material is $20 \mathrm{~mJ} / \mathrm{cm}^{2}$ for a $\Delta n_{\max }=1.7 \times 10^{-3}$. In comparison, for a thick sample of $\mathrm{BaTiO}_{3}{ }^{7}$ the experimentally measured saturation exposure is approximately $100 \mathrm{~mJ} / \mathrm{cm}^{2}$. Recent experiments with thin samples of $\mathrm{BaTiO}_{3}{ }^{8}$ and doped $\mathrm{SBN}^{9}$ indicate sensitivities and $\Delta n$ roughly equivalent to the photopolymer.

Figure 7 shows the effect of extending the total run time by increasing the time between holograms from $10 \mathrm{~s}$, as shown in Fig. 4, to $1 \mathrm{~min}$. As the exposure time increases, the dynamic range of the material is also used up as the residual monomer is polymerized. The decrease in hologram strength owing to loss of dynamic range is evident in Fig. 7. Therefore, not only are angular selectivity and exposure time important parameters for recording, but total run time is also. In order to maximize the number of holograms that can be stored, the total run time $\left(T_{\text {run }}\right)$ should be minimized. $T_{\text {run }}$ depends on $t_{\text {setup }}$ (the time to present an image to the system and to change the angle) and on $t_{e}$ (the exposure time), which varies as $t_{0} / N$, where $t_{0}$ is the single hologram saturation time and $N$ is the number of holograms. $T_{\text {run }}$ can be written as

$$
T_{\text {run }}=t_{\text {setup }} * N+t_{e} * N=t_{\text {setup }} * N+t_{0} .
$$

Using $N=1000, t_{\text {setup }}=0.1 \mathrm{~s}$, and $t_{0}=10 \mathrm{~s}$, we obtain a total run time of $110 \mathrm{~s}$. For a large number of holograms the setup time dominates $t_{0}$. However, since $t_{\text {setup }}$ can be equal to $0.1 \mathrm{~s}$ or less, the total run time even for 1000 holograms is below the $120 \mathrm{~s}$ that was experimentally demonstrated. Therefore, in practice the total run time is not the limiting factor in the number of holograms that can be stored.

The factor that does limit the number of holograms that can be stored is the thickness of the current samples. Increasing the thickness of the material

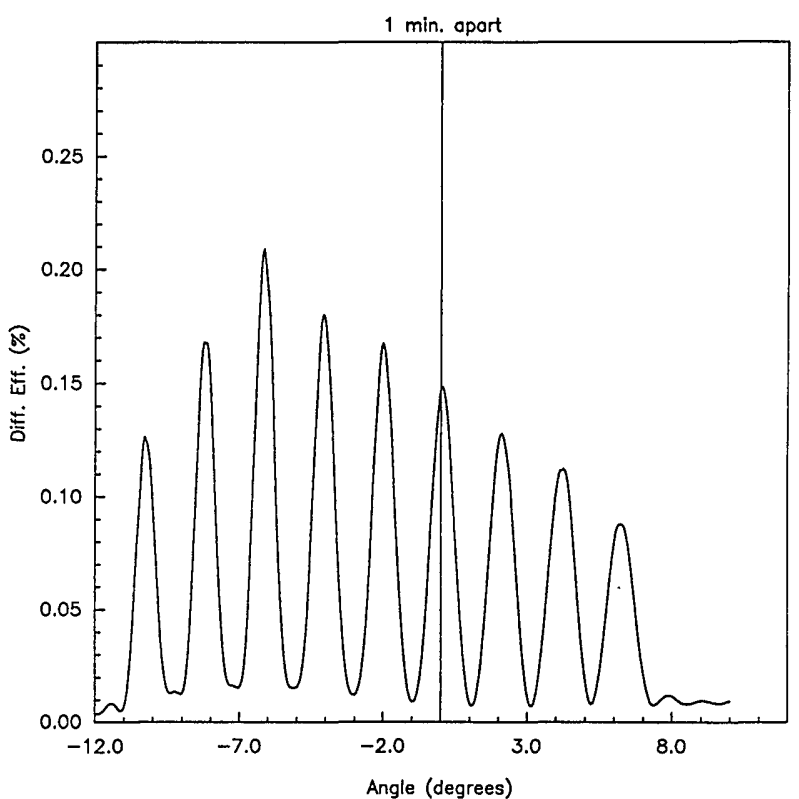

Fig. 7. Ten image plane holograms, 1 min. apart. 
increases the angular selectivity and hence the storage capacity of the material. We are currently investigating methods for fabricating thick samples of the HRF-150 material. Scattering may eventually limit the maximum useful thickness of the material. As an example, let us assume that we can fabricate material that is $4 \mathrm{~mm}$ thick with a sufficiently low scatter level. Then, using the method presented in Ref. 10 and with a maximum index perturbation of $1 \times 10^{-3}$, we predict that approximately 2,800 holograms, each with $0.01 \%$ diffraction efficiency, can be superimposed in this material. However, fabricating thick samples with good optical quality is not going to be trivial.

\section{Conclusions}

High-quality multiple holographic storage has been demonstrated in DuPont HRF-150 photopolymer. Angular multiplexing qualities have been shown to be predictable by using Kogelnik's two-wave diffraction theory. In addition, a pre-illumination pulse can be used to record equal strength holograms with the same exposure energy per hologram. Uniform diffraction efficiency for multiple holograms over a long run time can be obtained at the expense of hologram strength owing to the finite dynamic range of the material. Therefore total run time for sequential exposures is an important recording parameter and needs to be minimized. The thickness of the material is currently the limiting factor in the number of holograms that can be stored. With a large dynamic range, if it is possible to increase the thickness of the material without significantly degrading the optical quality, it might be possible for thousands of permanent holograms to be recorded with adequate diffraction efficiency.

The authors gratefully acknowledge Northrop's support for this work. K. Curtis acknowledges the
Northrop Fellowship for supporting his Ph.D. studies at the California Institute of Technology. The photopolymer was graciously made available to us by DuPont; in particular, the authors wish to thank W. Gambogi, Jr., K. Doraiswamy, and J. Calio of DuPont. In addition, we appreciate helpful discussions with J. Hong and S. Li.

\section{References}

1. F. H. Mok, M. C. Tackitt, and H. M. Stoll, "Storage of 500 high resolution holograms in a $\mathrm{LiNbO}_{3}$ crystal," Opt. Lett. 16, 605-607 (1991).

2. F. H. Mok, M. C. Tackitt, and H. M. Stoll, "A massively parallel optical template matcher/correlator," presented at the Optical Society of America Conference on Pattern Recognition, Boston, Mass., 4 November 1990.

3. D. Psaltis, D. Brady, X. G. Gu, and S. Lin, "Holography in artificial neural networks," Nature (London) 343, 325-330 (1990).

4. W. K. Smothers, T. J. Trout, A. M. Weber, and D. J. Mickish, "Hologram recording in DuPont's new photopolymer material," presented at the Second International Conference on Holographic Systems, Bath, UK, 13 November 1989.

5. H. Kogelnik, "Coupled wave theory for thick hologram gratings," Bell Syst. Tech. J. 48, 2909-2947 (1969).

6. W. S. Colburn and K. A. Haines, "Volume hologram formation in photopolymer materials," Appl. Opt. 10, 1636-1641 (1971).

7. J. Feinberg, D. Heiman, A. R. Tanguay, Jr., and R. W. Hellwarth, "Photorefractive effects and light-induced charge migration in barium titanate," J. Appl. Phys. 51, 1297-1305 (1980).

8. M. H. Garrett, J. Y. Chang, H. P. Jenssen, and C. Warde, "High photorefractive sensitivity in an $n$-type $45^{\circ}$-cut $\mathrm{BaTiO}_{3}$ crystal," Opt. Lett. 17, 103-105 (1992).

9. R. A. Vazquez, F. R. Vachss, R. R. Neurgaonkar, and M. D. Ewbank, "Large photorefractive coupling coefficient in a thin cerium-doped strontium barium niobate crystal," J. Opt. Soc. Am. B 8, 1932-1941 (1991).

10. J. Hong, P. Yeh, D. Psaltis, and D. Brady, "Diffraction efficiency of strong volume holograms," Opt. Lett. 15, 344346 (1990) 